\title{
CORRIGENDUM
}

\section{Evaluation of predictive tests for screening for dihydropyrimidine dehydrogenase deficiency}

MC van Staveren, H Jan Guchelaar, ABP van Kuilenburg, H Gelderblom and JG Maring

The Pharmacogenomics Journal (2014) 14, 400; doi:10.1038/tpj.2014.25

Correction to: The Pharmacogenomics Journal (2013) 13,389-395. The second author's name was misspelled in this article. The correct spelling is HJ Guchelaar. 